\title{
Progress on New Approaches to Old Ideas: Orbital-free Density Functionals
}

\author{
Valentin V. Karasiev, Debajit Chakraborty, and S.B. Trickey \\ 16 September 2013; chapter for Many-Electron Approaches in Physics, Chemistry and \\ Mathematics: A Multidisciplinary View, L. Delle Site and V. Bach eds., Springer Verlag (C)
}

\begin{abstract}
State-of-the-art treatment of nuclei and electrons in materials uses $a b$ initio molecular dynamics for nuclear motion driven by Born-Oppenheimer forces from the electrons. Almost universally, those forces are calculated from density functional theory in the Kohn-Sham form. The computational costs of the conventional KS implementation scale at least as the cube of the number of electrons. This is a formidable barrier to complex system simulations with long MD runs on department-scale machines, since the DFT force calculation dominates the per step cost. The difficulty arises from the explicit dependence of the non-interacting kinetic energy on the KS non-interacting orbitals. The cost scaling worsens with use of explicitly orbital-dependent exchange-correlation functionals are used. The alternative approach, use of DFT in its basic form, dates to Thomas-Fermi-Dirac theory. The challenge is to have sufficiently accurate orbital-free expressions for the KS kinetic energy and exchange-correlation functionals. We discuss progress on these tasks via constraint-based methods, with emphasis on developments since the Sept. 2010 "New Approaches to Many-Electron Theory" meeting.
\end{abstract}

\section{Motivation: Modern Relevance of the Old Idea}

Recent years have seen density functional theory (DFT) [1-3] emerge as the dominant theoretical framework for computing the electronic structure of materials and molecules. In our title, the "old idea" refers to the form of DFT from the dawn of quantum mechanics, Thomas-Fermi-Dirac (TFD) theory [4-10]. The conceptual framework of TFD is direct manipulation of a functional of the electron number density $n(\mathbf{r})$, without explicit reference to wave functions (many-body or noninteracting). In most circumstances, TFD is so oversimplified that it does not provide predictive treatment of materials properties. As a result, the pure DFT conceptual framework of TFD largely has been supplanted by use of the eigenfunctions and eigenvalues of the auxiliary Kohn-Sham (KS) system [11]. At the outset we must make a case, therefore, for any new approaches to the old idea.

A key example is the equation of state (EOS), which determines many materials properties crucial to both basic understanding and technological utilization. Often,

http://www.qtp.ufl.edu/ofdft

Quantum Theory Project, Departments of Physics and of Chemistry,

P.O. Box 118435, University of Florida, Gainesville FL 32611-8435 
the zero-temperature EOS and crystalline phase transitions along it can be handled quite accurately with modern DFT alone $[12,13]$. Beyond that, at ambient and higher temperatures, what is called $a b$ initio MD (AIMD; see [14-18]) generally handles the nuclear (or ionic) degrees of freedom well. In the Born-Oppenheimer approximation, AIMD with DFT receives the electronic forces on the nuclei from a DFT calculation done at each configuration of nuclei. Thus, one expects AIMD to have the advantage of explicit quantum mechanical treatment of the electrons combined with the essentially classical contributions of the nuclei.

The computational cost problem comes from having a $\mathrm{KS}$ calculation at every MD step. Compared to the cost per conventional MD step, KS calculations are quite expensive (typically $\geq 98 \%$ of the computational cost of each AIMD step) and scale badly with system size. A seemingly unrelated fact is that, because DFT is rooted in existence theorems [1-3], computation requires approximate functionals for the exchange $(\mathrm{X})$ and correlation $(\mathrm{C})$ contributions. In reality, these two facts are deeply intertwined as a challenge to DFT-AIMD as a basic tool of materials research. Somehow the accuracy and range of applicability of approximate functionals must be balanced with control of computational cost (see review of algorithms and their performance in the chapter by Tzanov and Tuckerman).

The problem originates in the DFT variational minimization via the KS decomposition. That yields (details below) an eigenvalue problem for the auxiliary KS (non-interacting) many-Fermion system at the physical density, $n(\mathbf{r})$ [11], to wit

$$
h_{K S}[n] \varphi_{i}=\varepsilon_{i} \varphi_{i} \quad, \quad n(\mathbf{r})=\sum_{i} f_{i}\left|\varphi_{i}(\mathbf{r})\right|^{2} .
$$

Here the $f_{i}$ are occupation numbers, $f_{i}=0,1,2$ (for the non-spin-polarized case) $[19,20]$. Diagonalization implicit in the KS eigenvalue problem is the computational bottleneck. Even with the simplest exchange-correlation (XC) functional (no explicit orbital dependence), KS calculations scale in principle no better than $N_{e}^{3}$, with $N_{e}$ being total number of electrons. AIMD driven by KS DFT therefore also scales as $N_{e}^{3}$ or worse, per MD step.

Unhappily for balancing cost and accuracy, much of the recent progress on better $\mathrm{XC}$ approximations worsens that scaling. Though the basic DFT theorems [1-3] do not invoke the KS orbitals and eigenvalues, most effort today on better XC functionals involves DFT exact exchange. That has the same form as in the HartreeFock approximation but with the KS orbitals. Some representative references include [21-34]. All that is important here is that DFT exact exchange, whether by itself or in so-called hybrid functionals (mixtures of exact exchange and explicit density functionals), adds an explicit orbital dependence that worsens the KS cost scaling, typically to $N_{e}^{4}$ or more [35].

The deep irony, of course, is that decomposition of the fundamental density functional into non-interacting $(\mathrm{KS})$ and remainder parts does not require explicit use of the KS orbitals. In addition to defining the exact X energy and giving the density, the $\mathrm{KS}$ orbitals yield the major part of the electronic kinetic energy (KE). That eliminates the need for an explicit KE functional $T[n]$. But such an explicit functional was precisely the essence of the "old idea" [4-10]. Pursuit of the old idea never entirely 
died out, but its modern use in AIMD seems to have begun with the implementation of TF AIMD by Clerouin, Pollock and Zerah [36]. That was preceded by the formulation of a non-local KE functional by Chacón, Alvarellos, and Tarazona [37] and, a few years later, the AIMD-oriented functionals by Wang and Teter [38] and Smargiassi, Madden, and collaborators [39-41] and other innovations, e.g. [42]. The approach has come to be known as orbital-free DFT (OFDFT), though it might be as insightful to call it one-orbital DFT.

In what follows, we summarize OFDFT basics and establish notation, then discuss progress since the 2010 "New Approaches to Many-electron Theory" (NAMET) meeting, with a focus on priority issues for our research program. This involves improvements in both orbital-free kinetic energy (OFKE) and XC functionals. Much of our work is on orbital-free, free-energy density functional approximations for systems at non-zero temperature. A recent review is [43]. Note that sometimes, particularly for $T>0 \mathrm{~K}$ calculations, the term "OFDFT" is used in the literature to be synonymous with a Thomas-Fermi or Thomas-Fermi-von Weizsäcker type KE functional. One must be alert to this overly restrictive usage. Also note that we do not treat the embedding form of OF-DFT $[44,45]$. The distinction is important: requirements on OF-KE embedding functionals differ substantially from those on approximations for the KS KE functionals on which we work.

\section{Basics}

\section{$2.1 \mathrm{DFT}$}

To be reasonably self-contained, we summarize basic DFT in non-spin-polarized form. The spin-polarized extension is generally straightforward. The foundational DFT theorems demonstrate the existence of a functional of the density alone,

$$
\mathscr{E}[n]=T[n]+U_{e e}[n],
$$

which is independent of the external potential on the electrons (or "universal"). Its contributions are the total KE and total Coulomb energy, respectively. In combination with the external energy $E_{\text {ext }}[n]$, it has the variational property

$$
\min _{n}\left\{\mathscr{E}[n]+E_{\text {ext }}[n]\right\}=E_{0}\left[n_{0}\right],
$$

where $E_{0}$ and $n_{0}$ are the ground state total energy and density respectively, and $\int d \mathbf{r} n(\mathbf{r})=N_{e}$. For AIMD $E_{e x t}[n]$ comes from the interaction with nuclei of charge $Z_{\alpha}$ situated at sites $\mathbf{R}_{\alpha}$,

$$
E_{\text {ext }}[n]=-\sum_{\alpha} Z_{\alpha} \int d \mathbf{r} \frac{n(\mathbf{r})}{\left|\mathbf{r}-\mathbf{R}_{\alpha}\right|} .
$$

(We use Hartree a.u. unless noted otherwise.) To approximate $\mathscr{E}[n]$ and make the variational minimization feasible, KS considered an auxiliary system of noninteracting fermions with the same density as the physical one. That system has 
eigenfunctions and density given by Eqs. (1). From those, one can define the KS KE $T_{s}$ and $\mathrm{X}$ energy $E_{x}$, then rearrange the universal functional (2) as

$$
\begin{aligned}
\mathscr{E}[n] & =T_{s}[n]+E_{H}[n]+E_{x}[n]+\left\{U_{e e}[n]-E_{H}[n]-E_{x}[n]+T[n]-T_{s}[n]\right\} \\
& :=T_{s}[n]+E_{H}[n]+E_{x}[n]+E_{c}[n] .
\end{aligned}
$$

In order, the quantities are

and

$$
\begin{gathered}
E_{H}[n]:=\frac{1}{2} \int d \mathbf{r}_{1} d \mathbf{r}_{2} \frac{n\left(\mathbf{r}_{1}\right) n\left(\mathbf{r}_{2}\right)}{\left|\mathbf{r}_{1}-\mathbf{r}_{2}\right|}, \\
E_{x}[n]:=\left\langle\Phi_{s}[n]\left|\hat{V}_{e e}\right| \Phi_{S}[n]\right\rangle-E_{H}[n],
\end{gathered}
$$

$$
T_{s}\left[\left\{\varphi_{i}\right\}\right]=\frac{1}{2} \sum_{i=1}^{N_{\mathrm{e}}} f_{i} \int d \mathbf{r}\left|\nabla \phi_{i}(\mathbf{r})\right|^{2}:=\int d \mathbf{r} t_{\text {orb }}[n(\mathbf{r})] .
$$

The KS Slater determinant is $\Phi_{s}[n] ; \hat{V}_{e e}$ is the electron-electron repulsion potential.

Observe that in KS-DFT the interacting part of the KE, $T-T_{S}$, is put into the C functional $E_{c}$ and that $T_{s}[n] \leq T[n]$ by definition $\left(T_{s}[n]\right.$ is the minimum Fermion expectation of the $N_{e} \mathrm{KE}$ for the specified $n$ ). Note also that in (8) we have dropped a usually inconsequential surface term relative to the more familiar KS KE definition in terms of the Laplacian of the orbitals.

The potential in the KS equation (1) is

$$
\begin{aligned}
\mathrm{v}_{K S} & =\mathrm{v}_{H}+\mathrm{v}_{\text {ext }}+\mathrm{v}_{x c} \\
\mathrm{v}_{H}=\int d \mathbf{r}_{2} \frac{n\left(\mathbf{r}_{2}\right)}{\left|\mathbf{r}-\mathbf{r}_{2}\right|}, \mathrm{v}_{e x t} & =-\sum_{\alpha} \frac{Z_{\alpha}}{\left|\mathbf{r}-\mathbf{R}_{\alpha}\right|}, \mathrm{v}_{x c}=\frac{\delta E_{x c}}{\delta n} .
\end{aligned}
$$

In the foregoing expressions, the dependence of the density and of the total energy upon the nuclear coordinates $\{\mathbf{R}\}$ has been suppressed. With that in mind, the electronic force on a given nucleus is $-\nabla_{\mathbf{R}_{\alpha}}\left(\mathscr{E}+E_{\text {ext }}+E_{\text {ion-ion }}\right)$. Here $E_{\text {ion-ion }}(\{\mathbf{R}\})$ is the inter-nuclear repulsion energy.

\subsection{Orbital-free Functional Framework}

Until exact and hybrid X functionals (the third rung of the Perdew-Schmidt XC functional ladder [26]) became popular, approximate XC functionals used in materials simulations were orbital-free, though not usually labeled as such. Even now, the most widely used XC functionals, PBE [46] and the local density approximation (LDA) [47], are orbital-free. Generically they are

$$
E_{x c}=\int d \mathbf{r} \mathrm{e}_{x c}[n(\mathbf{r}), \nabla n, \ldots] .
$$

Dependence of $\mathrm{XC}$ approximations upon density derivatives beyond gradients is not a focus here. See, however, recent work by Cancio et al. [48]. 
Assuming the use of an OFXC functional, only $T_{s}$ has explicit orbital dependence. Thus, we seek OF approximations of the form

$$
T_{s}[n]=\int d \mathbf{r} t_{s}[n(\mathbf{r}), \nabla n, \ldots],
$$

where the exact result would match Eq. (8), $t_{s}[n]=t_{\text {orb }}[n]$. A critical reader may wonder, why not use $T[n]$ rather than $T_{s}[n]$ ? At least two responses are relevant. All of the roughly 40 years of effort on better XC functionals has been in the KS context. Indeed, the exact $E_{X}[n]$ in DFT is defined in terms of the KS decomposition, hence so is $E_{c}$. Another motivation is the Coulomb virial theorem, $E_{0}\left[n_{0}\right]=-T\left[n_{0}\right]$. This suggests that finding a useful approximation for the full $T[n]$ might be tantamount to finding an approximation for the universal functional $\mathscr{E}[n]$ [49].

With OF approximations for $T_{s}$ and $E_{x c}$, variation with respect to the density yields the OF Euler-Lagrange equation

$$
\frac{\delta T_{s}}{\delta n}+\frac{\delta E_{H}}{\delta n}+\frac{\delta E_{x c}}{\delta n}+\mathrm{v}_{e x t} \equiv \frac{\delta T_{s}}{\delta n}+\mathrm{v}_{K S}=\mu .
$$

Here $\mu$ is the electron chemical potential.

\subsection{Single-point Approximate KE Functionals}

The simplest KE approximation would seem to be Thomas-Fermi,

$$
T_{S}[n] \approx T_{T F}[n]:=\frac{3}{10}\left(3 \pi^{2}\right)^{2 / 3} \int d \mathbf{r} n^{5 / 3}(\mathbf{r}) \equiv c_{T F} \int d \mathbf{r} n^{5 / 3}(\mathbf{r}) .
$$

But this is not a good starting approximation, despite its widespread use as such. The point is that the von Weizsäcker KE [8],

$$
T_{W}[n]:=\frac{1}{8} \int d \mathbf{r} \frac{|\nabla n(\mathbf{r})|^{2}}{n(\mathbf{r})},
$$

is both a lower bound to the KS KE [50-53],

$$
T_{W}[n] \leq T_{s}[n],
$$

and is exact for one electron and for a two-electron singlet. One immediately sees that $T_{T F}$ cannot be correct in the tail region of a system as simple as a many-electron atom, since the ionization potential theorem [54] shows that tail to be one-electronlike. These physical arguments lead to adoption of the Pauli-term decomposition

$$
T_{S}[n]=T_{W}[n]+T_{\theta}[n], \quad T_{\theta}[n] \geq 0 .
$$

Non-negativity of the Pauli term $T_{\theta}$ is a rigorous requirement [55-59], as is the more demanding requirement on the corresponding potential, 


$$
\mathrm{v}_{\theta}:=\frac{\delta T_{\theta}[n]}{\delta n(\mathbf{r})} \geq 0 \quad, \forall \mathbf{r}
$$

By invoking this pair of requirements, we have made two choices regarding approximate functional development. The first is to work on one-point functionals, generically $\mathscr{F}[n]=\int d \mathbf{r} f[n, \nabla n \ldots]$ rather than two-point functionals. A two-point or non-local OF-KE functional has the generic form

$$
T_{\text {nonloc }}[n]=\int d \mathbf{r}_{1} d \mathbf{r}_{2} n^{\lambda}\left(\mathbf{r}_{1}\right) K\left[n\left(\mathbf{r}_{1}\right), n\left(\mathbf{r}_{2}\right), \mathbf{r}_{1}, \mathbf{r}_{2}\right] n^{\gamma}\left(\mathbf{r}_{2}\right)
$$

with $\lambda+\gamma=8 / 3$ for a dimensionless kernel $K$. The kernel typically is constrained to recovery of the homogeneous electron gas response. Commonly a form such as (18) is used in conjunction with $T_{T F}+T_{W}$. An accessible motivation for the non-local part is in [60]. Essentially the goal is to generate atomic and molecular electronic shell effects in the density and corresponding Friedel oscillations in solids. While there is clear progress on this formulation, there are problems which we prefer to sidestep. For one, different approximate kernels so far are needed for semiconductors, covalent systems, and metals [61-63]. The universality of DFT thus is lost. The six-dimensional form of the kernels also sacrifices some of the OFDFT computational cost advantage (e.g. remarks in [64]). Conceptually, the linear response formulation implicitly requires a reference uniform density, an ill-defined quantity for any un-enclosed system (an atom or molecule). And, there are non-linear instabilities with the simplest kernels (called density-independent) [65], which forces use of more intricate, density-dependent kernels.

Secondly, we eschew heavily parametrized functionals. Just as with the XC functional, no constructive route (e.g. perturbation expansion) to approximate KE functionals is provided in the proofs of the DFT theorems $[1-3,11]$. An option to surmount that obstacle is parametrization to a large data set, as in the M-06L XC functional [66], for example. But that approach inherently has limited predictive capability and limited insight into the physics omitted from the functional. The state of the art for non-empirical functional construction use of scaling relations, bounds, asymptotics, and limiting case behavior as constraints on allowable functional forms and parameter values. Such functionals are called constraint-based.

The third author's 2010 NAMET talk summarized our work (with collaborators) on constraint-based OFKE functionals up to and through [67]. Earlier papers are [68-70]. That talk also summarized constraint-based OF XC functional work in [71] and subsequently in [72]. What follows picks up from there in our own work and touches on work which has influenced us and our collaborators.

\section{Progress}

\subsection{Non-empirical KE Functional Parametrization}

The first step past the LDA is to include density gradients. But the second-order gradient approximation (SGA) is a flawed OFKE functional; see [67]. An analo- 
gous problem in the development of $\mathrm{X}$ functionals led to construction of generalized gradient approximations (GGAs). The GGA form for the OF KE is

$$
T_{s}^{G G A}[n]=c_{T F} \int d \mathbf{r} n^{5 / 3}(\mathbf{r}) F_{t}(s(\mathbf{r})) .
$$

The enhancement factor $F_{t}$ depends on the dimensionless reduced density gradient

$$
s:=\frac{1}{2\left(3 \pi^{2}\right)^{1 / 3}} \frac{|\nabla n|}{n^{4 / 3}}
$$

From the form of $T_{W}$, the enhancement factor for $T_{\theta}^{G G A}$ readily is found to be

$$
F_{\theta}(s)=F_{t}(s)-\frac{5}{3} s^{2} .
$$

Eqs. (11), (14), and (16) also yield

$$
T_{\theta}[n]:=\int d \mathbf{r} t_{\theta}[n], \quad t_{\theta}=t_{s}-\frac{1}{8} \frac{|\nabla n|^{2}}{n} .
$$

(Note that Eq. (9) of [67] should read $t_{\theta}=t_{\text {orb }}+\frac{1}{2} \sqrt{n} \nabla^{2} \sqrt{n}$ because $t_{\text {orb }}$ as defined there differs from Eq. (8), as well as a sign error. The different definition means that in [67] $t_{s}$ includes a $\nabla^{2} n$ term. The outcomes are unchanged.) Positivity of both $T_{\theta}$ and $t_{\theta}[53,54,58]$ means that the GGA enhancement factor must obey

$$
F_{\theta}(s(\mathbf{r})) \geq 0, \forall \mathbf{r}
$$

Remarks: This is a much stronger constraint than those found in X GGA development. Second, Levy and Ou-Yang [58] arrive at $t_{\theta}>0$ by the equality of two integrals. This involves the usual issue of energy densities, namely, omission of any terms which would integrate to zero.

Two forms of generalized gradient approximation (GGA) for the OFKE were treated in $[67,68]$. The relevant one here is

$$
F_{t}^{P B E v}(s)=1+\sum_{i=1}^{v-1} C_{i}\left[\frac{s^{2}}{1+a_{1} s^{2}}\right]^{i}, v=2,3,4 .
$$

(The label "PBE" is solely because for $v=2$ it has the same form as the PBE $\mathrm{X}$ functional enhancement factor [46].) Those papers involved calibration of the parameters $a_{i}$ and $C_{i}$ via matching the KS energy surface shape on very small training sets (one to three molecules) rather than matching KE values. That calibration fixes the main problem of prior GGA KE functionals such as from [73-77], namely violation of the Pauli potential non-negativity constraint Eq. (17) at nuclear sites. Respect for that constraint was shown in $[67,68,78,79]$ to be responsible for correct description of attractive forces, hence for prediction of molecular and solid binding. The price is a strong overestimation of the KS KE. Though the PBE2 functional proved to be surprisingly transferable, the parametrization is not compliant with constraint- 
based development. (The PBE2 functional is not positive definite everywhere, but the violations are in regions which are unimportant in practice.)

Recently we have devised a procedure for non-empirical calibration of a somewhat more flexible form of the enhancement factor [80]. We impose three requirements: (a) recovery of the second-order gradient expansion (GE) for $s<<1$ [81], hence

$$
F_{t}(s)=1+(5 / 27) s^{2}+O\left(s^{4}\right),
$$

(b) non-negativity of the Pauli potential $\mathrm{v}_{\theta}$ as per Eq.(17), and

(c) recovery of $\mathrm{vW}$ behavior in the large-s limit,

$$
\lim _{s \rightarrow \infty} F_{\theta}(s)=0 .
$$

This last constraint follows from the character of charge densities far from any nucleus and the IP theorem [54]. The form in Eq. (24) cannot meet all three constraints, so we adopted a form which resembles the VT $\{84\}$ X enhancement factor [72],

$$
F_{t}^{V T 84 F}(s)=1-\frac{\mu s^{2} e^{-\alpha s^{2}}}{1+\mu s^{2}}+\left(1-e^{-\alpha s^{m / 2}}\right)\left(s^{-n / 2}-1\right)+\frac{5}{3} s^{2},
$$

with $m=8, n=4$. (The "F" in VT84F simply denotes the origin of this functional as the zero-temperature limit of a new non-interacting free energy functional.) The next-to-last term in Eq. (27) meets constraint (c), so the parameters $\mu$ and $\alpha$ must follow from constraints (a) and (b). The small-s expansion gives

$$
F_{t}^{V T 84 F}(s)=1+(5 / 3+\alpha-\mu) s^{2}+O\left(s^{4}\right)
$$

whence constraint (a) yields $\alpha=\mu-5 / 3+5 / 27$. Evaluation of the Pauli potential for small- $r$ (from a nuclear site) from the Kato nuclear-cusp condition [82] density

$$
n(r) \sim e^{-2 Z r}=(1-2 Z r)+O\left(r^{2}\right) .
$$

gives $\mathrm{v}_{\theta}^{G G A}(r) \sim a / r$ [67] where $a$ is a constant which depends on the specific enhancement factor. For the enhancement factor Eq. (27), the singular term $a / r$ becomes marginally positive for $\mu=2.778$. That gives $\alpha=1.2965$. Note that while we have made several design choices, there is no calibration to experiment or to KS calculations. Initial results from this non-empirical functional are quite encouraging, see Subsection 3.3 below.

\subsection{OF XC Functionals}

We already have emphasized the critical importance of better orbital-free XC functionals $E_{x c}[n]$, even though that is not the focus of most present-day XC functional development. Work in [72,83-85] shows ways to make significant improvements in GGA $E_{x}$ energetics. In addition, that collaboration has presented both an improved (relative to B3LYP [22] and revTPSS [86]) meta-GGA and an improved hybrid X functional. One of these developments, the PBEmol X functional [84] is interesting 
both because of what it illustrates about the challenges of better OF XC functionals and a challenge it brings into play for GGA OFKE functionals. We discuss the second item below. Here we sketch the parametrization of the PBEmol X functional.

For the exact exchange functional, self-exchange must cancel self-repulsion. For the Hydrogen atom density

$$
n_{1}(r)=\pi^{-1} \exp (-2|\mathbf{r}|)
$$

this means

$$
E_{H}\left[n_{1}\right]+E_{x}\left[n_{1}\right]=0
$$

Furthermore, self-correlation must vanish in a one-electron system, so

$$
E_{c}\left[n_{1}\right]=0 .
$$

However, most approximate XC functionals have neither behavior.

The PBEmol X functional re-parametrizes the Perdew-Burke-Ernzerhoff PBE $\mathrm{X}[46]$

$$
\begin{aligned}
E_{x}^{G G A}[n] & =C_{x} \int d \mathbf{r} n^{4 / 3}(\mathbf{r}) F_{x}^{G G A}(s) \\
C_{x} & :=-\frac{3}{4}\left(\frac{3}{\pi}\right)^{1 / 3} \cdot \\
F_{x}^{P B E}(s) & :=1+\kappa-\frac{\kappa}{1+\mu s^{2} / \kappa} .
\end{aligned}
$$

to satisfy (31). The constant $\kappa=0.804$ is set to satisfy the Lieb-Oxford bound [87] and is not at issue here. For PBE, $\mu_{P B E}=0.21951$, chosen to recover LDA linear response (by combination with the PBE $\mathrm{C}$ functional). A revised parametrization, called PBEsol [88], uses the gradient expansion value, $\mu_{G E}=10 / 81$, to improve the quality of calculated surface and crystal properties, but at the cost of lessened accuracy for finite systems. The PBEmol parametrization goes in the other direction. Enforcement of Eq. (31) yields $\mu_{P B E m o l}=0.27583$. This forces a shift in the $\beta$ parameter which appears in the PBE C functional: $\beta_{P B E m o l}=0.08384$ versus $\beta_{P B E}=$ 0.066725 versus $\beta_{P B E s o l}=0.046$. The result is beneficial in the sense of coming closer to satisfying Eq. (32),

$$
\begin{aligned}
E_{c, \text { PBEmol }}\left[n_{1}\right] & =-0.004876 \text { hartree } \\
E_{c, \text { PBE }}\left[n_{1}\right] & =-0.005976 \text { hartree } .
\end{aligned}
$$

However, as expected from the shift downward in $\mu$ from PBE to PBEsol to improve extended system results, the shift upward in PBEmol improves most (but not all) calculated molecular quantities but worsens the extended system ones. The PBE form is just too restrictive. See [89], especially the closing remark. 


\subsection{Solution of the OFDFT Euler-Lagrange Equation and Local Pseudopotentials}

Eq. (16) combined with $\delta T_{W} / \delta n=-\frac{1}{2} n^{-1 / 2} \nabla^{2} n^{1 / 2}$ converts the Euler-Lagrange equation (12) into a one-orbital modified KS equation,

$$
\left\{-\frac{1}{2} \nabla^{2}+\frac{\delta T_{\theta}}{\delta n}+\mathrm{v}_{K S}\right\} n^{1 / 2}=\mu n^{1 / 2} .
$$

This is the form which suggests that OFDFT might well be called one-orbital DFT. It also is strongly suggestive that solution would be straightforward with a standard KS code. At the time of the NAMET meeting, two of us (VVK and SBT) had just confirmed and extended [78] the antecedent finding by Chan, Cohen, and Handy [90] that this appealing idea fails. The problem is the peculiar potential added to the ordinary $\mathrm{v}_{K S}$ in Eq. (36). Those GGA OFKE functionals with proper $\delta T_{\theta} / \delta n \equiv \mathrm{v}_{\theta} \geq 0$ exacerbate the trouble because of their inescapable positive nuclear site singularities; recall the discussion at Eq. (29) above. Direct minimization algorithms, such as in the PROFESS code discussed below, are essential.

First, however, we deal with numerical issues related to the potential itself. Many codes for solving the KS equations in periodic systems use a plane-wave basis and pseudopotentials (PPs). We include projector augmented wave (PAW) methodology somewhat loosely in the PP category. For driving AIMD calculations, plane-wavePP approaches have a large advantage over methods which use nuclear-site-centered basis sets. Such basis sets generate so-called Pulay forces whereas a plane-wave basis does not. Though OFDFT in principle is an all-electron method, efficient implementation of Fourier space methods, including plane wave basis sets, requires regularization of the nuclear-electron interaction singularity. The challenge then is the dominance of non-local, i.e., orbital-dependent, PPs, which clearly are inapplicable in OF-DFT calculations. OFDFT requires local pseudopotentials (LPPs).

In the absence of normative practice for developing such LPPs for single-point functionals, we devised a new method [78]. The LPP is constructed as a normalized linear combination of angular momentum components ( $\ell$-components) of a normconserving non-local PP (NLPP). The linear coefficients are determined by requiring that the LPP reproduce some bulk property as given by the parent NLPP, for example, the equilibrium lattice parameter of a solid. Calibration to an equilibrium property does not, of course, guarantee that the LPP will be reliable for treating crystalline phase transitions. We investigated this issue for $\mathrm{Li}$ [91] by comparison to all-electron results for clusters of the same local symmetry and near-neighbor distances. Both ordinary norm-conserving PPs and projector-augmented wave (PAW) data sets used with codes such as ABINIT [92, 93], QuANTUM ESPRESSO [94], and VASP [95-98] turn out to have relatively small reliable compression ranges. Depending on detailed criteria, maximum compressions (with respect to ambient density) of 1.5, 4.5, or 7 were found. Some PAW data sets include a so-called compensation charge density (CCD) contribution. We found it to be problematic at high compressions. Omission of the CCD and use of a cutoff radius essentially half the 
standard value, $r_{c}=0.8$ bohr vs. $1.61 \mathrm{bohr}$, gave Li PAW LDA and GGA data sets which are reliable to at least 140 -fold compression.

An alternative procedure, appropriate for simple cubic Hydrogen (sc-H), is to fit the parameters of the simple Heine-Abarenkov model $[99,100]$ such that a bulk property (e.g. equilibrium lattice constant) from a more sophisticated KS bareCoulomb potential calculation is reproduced. Transferability of such a model potential was tested up to more than 50-fold compression [101], a material density around $25 \mathrm{~g} / \mathrm{cm}^{3}$. The result is a cutoff radius $r_{c}=0.25$ bohr. Geng et al. [102] recently gave what they characterized as a hard PAW for Hydrogen, $r_{c}=0.794$ bohr. They justified this rather larger $r_{c}$ by comparison with an all-electron calculation of $E_{f c c}-E_{b c c}$ over the range $0 \leq P \leq 4 \mathrm{TPa}$. Since PAW data set transferability requires non-overlap of augmentation regions, one concludes that the Geng et al. PAW could be applicable to sc-H for a lattice constant no less than twice $r_{c}$, i.e. $1.588 \mathrm{bohr}$ or an $\mathrm{sc}-\mathrm{H}$ density up to about $3 \mathrm{~g} / \mathrm{cm}^{3}$. This is roughly six-fold compression as compared to 50-fold for the PAW of [101].

Most of our OFDFT calculations have used a locally modified version of the PROFESS code $[103,104]$. Originally for use with two-point OFKE functionals (recall brief discussion), PROFESS performs periodic OFDFT calculations. For orbital-free AIMD, we have built an interface between PROFESS and QUANTUM ESPRESSO [94] to utilize OFDFT forces in QUANTUM ESPRESSO [105]. As an example, we performed static lattice KS and OFDFT calculations with the TF, SGA, recently proposed non-empirical APBEK [106] GGA, and VT84F (recall Eq. (27)) OFKE functionals for sc-H. The left panel of Figure 1 compares KS and OFDFT total energies per atom as a function of sc lattice constant. The TF, SGA, and APBEK functionals all fail to produce binding. The new, non-empirical VT84F predicts binding with a moderately underestimated lattice constant (2.556 bohr vs. 2.773 bohr for KS, about 6\%) and a too-stiff bulk modulus (175.3 GPa vs. 108.4 $\mathrm{GPa}$ for KS) [101]. The right panel of Figure 1 shows the pressure for sc-H as a function of material density for the same set of functionals. At the lowest density, $\rho_{\mathrm{H}}=0.7 \mathrm{~g} / \mathrm{cm}^{3}$, the TF, SGA, and APBEK OFKE pressure errors are about $200 \%$. The VT84F functional underestimates by about $80 \%$. With increasing density, pressures from all the OFKE functionals approach the reference KS values, with VT84F pressures doing so most quickly.

\section{Some Interesting Issues}

\subsection{GGA Functional Form Limits}

As mentioned already, XC GGAs cannot meet all the constraints derivable for the exact $E_{x c}$. Analogously for the KS KE, Wang, Stott, and von Barth [107] have argued that the GGA form is so restrictive that a proper $T_{s}[n]$ cannot be obtained. Though the argument is worth study, we do not concur with the priority of constraints that they 

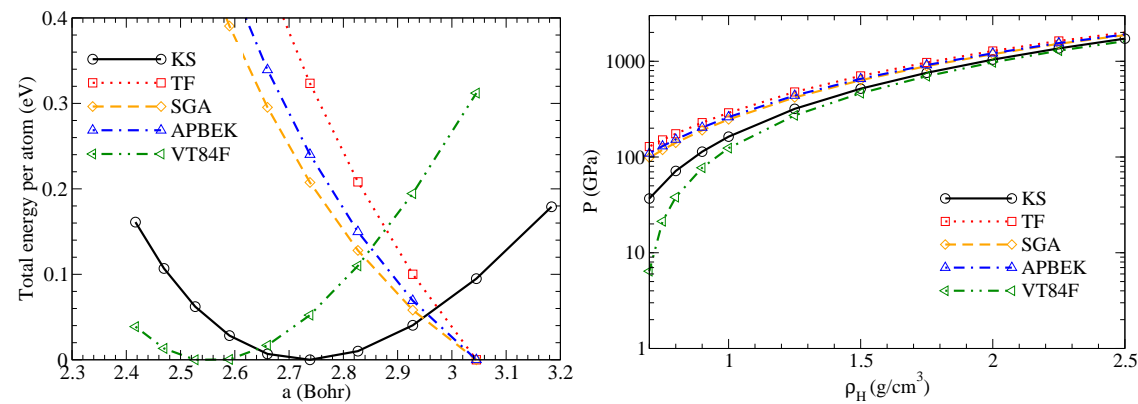

Fig. 1 Comparison of KS and various OFDFT functionals for sc-H. All with Perdew-Zunger (PZ) [47] LDA XC. Left: total energy per atom (shifted to zero) as a function of lattice constant. Right: Pressure as a function of material density.

choose. Specifically, requiring recovery of the gradient expansion seems unhelpful for systems with small $N_{e}$, the regime in which they tested.

In fact, that regime has a more severe problem. The H-atom density $n_{1}(r)$, Eq. (30), used for the PBEmol parametrization discussed above, illustrates the difficulty. Because $T_{W}\left[n_{1}\right]$ is exact, the exact $T_{\theta}$ must vanish, $T_{\theta}\left[n_{1}\right]=0$. But no GGA can do that and also satisfy $F_{\theta}$ positivity, Eq. (23) and the related $t_{\theta}$ positivity. The problem is not just with the GGA form. In essence, one is up against the requirement that Eq. (16) must be revised to read

$$
T_{S}[n]=T_{W}[n]+\left(1-\delta_{N_{e}, 1}\right)\left(1-\delta_{N_{e}, 2}\right) T_{\theta}[n] \quad, \quad T_{\theta}[n] \geq 0 .
$$

The notation is incomplete for simplicity, since the $\delta_{N_{e}, 2}$ applies only for $N_{e}=2$ singlets. It is very difficult to imagine being able to mimic this behavior in an approximate functional without introducing explicit number-dependence (and its attendant difficulties). This is an example of the explicit number-dependence issue first mentioned (we believe) by Lieb [3] as being an inescapable property of the universal density functional and discussed more recently in [108].

Another issue is one we have already encountered, namely, the singularity of a GGA Pauli potential at nuclear sites. At such sites, the exact Pauli potential $v_{\theta}$ calculated by inversion of the KS equations has positive, finite values [67]. The Pauli potential from the previously published GGA functionals mentioned in Subsection 3.1, among them APBEK, all have negative nuclear site singularities. Those functionals give reasonable values for the KS KE near equilibrium but they fail, in general, to predict molecular and solid binding. In contrast, the GGA functionals we have constructed, Eqs. (24) and (27), have positive nuclear site singularities. Those functionals do describe the energy surface shape reasonably well, hence predict at least qualitatively correct binding. But the KE they give is strongly overestimated.

Such nuclear site singularities of the Pauli potential seem to be an intrinsic property of GGA KE functionals. The singularities can be eliminated by use of dimensionless combinations of higher order derivatives (reduced density derivatives, RDDs). Definitions and examples are in [67]. The difficulty then is to find appro- 
priate functional forms for use of those RDDs. We have little or no insight about such forms at this point. There is also an issue of complicated functional derivatives arising from functionals that incorporate RDDs, but this may not be much different from what already is encountered in meta-GGA XC functionals [86].

\subsection{Fitness Tests for OFKE Approximations}

Many fitness tests of OFKE approximations have been devised. For years, perhaps the most common was to evaluate the atomic or molecular total energy or KE for an OFDFT expression with a KS-DFT or Hartree-Fock density as input $[109,110]$. Given our focus on AIMD-OFDFT, we have emphasized reproduction of KS binding. Specifically, given the KS solution (with a prescribed $E_{x c}$ ) for a molecule or solid, does a particular OFKE approximation reproduce the shape of the binding energy curve or curves (e.g. energy vs. bond length or lattice parameter) when fed the KS densities as input? If not, then there is no sense solving the Euler-Lagrange equation for that approximation, since it does not get the right answer (reproduce the KS result) when fed the right density (the KS density).

García-Aldea and Alvarellos [111] have developed and used a different fitness test. For a given approximate OFKE functional $T_{\text {approx }}$, they define

$$
T_{\text {approx }}[n]=\int d \mathbf{r} \mid t_{\text {approx }}[n(\mathbf{r}), \nabla n, \ldots] .
$$

analogously with Eq. (11). With that, they define a quality factor

$$
\sigma\left[T_{\text {approx }}\right]:=\frac{\int d \mathbf{r}\left|t_{s}[n]-t_{\text {approx }}[n]\right|}{T_{S}[n]} .
$$

This allows them to consider OFKE approximations which differ by Laplaciandependent terms that may, in general, alter $\delta T_{\text {approx }} / \delta n$ without altering the value $T_{\text {approx }}[n]$ delivered for a particular $n$. See their work in [112] as well as the work by Cancio et al. mentioned earlier [48]. In essence they consider the admixture

$$
t_{\text {approx }, \alpha}:=t_{\text {approx }}+\alpha \nabla^{2} n .
$$

In [111] $\alpha$ was optimized by minimizing $\sigma\left[T_{\text {approx }, \alpha}\right]$ over Slater-type-orbital atomic densities for the ten lightest atoms and the resulting quality factors for 21 OFKE functionals were compared. The remarkable conclusion is that TF, Eq. (13), augmented by a Laplacian term with $\alpha=0.167$, is best.

The difficulties with this outcome are evident. The TF functional suffers from the Teller non-binding theorem [9]. It is clearly wrong for $N_{e}=1$ and singlet $N_{e}=2$. And our calculations on simple cubic $\mathrm{H}$ [101] show that it gives total energy errors per atom of the order of $40 \%$ at roughly 4 -fold-compression and substantial pressure errors (factor of 2 or worse) up to about the same compression. Recall Fig. 1 and associated discussion above. We therefore are unconvinced that the quality factor Eq. (39) is useful in functional development. 


\subsection{Information Theoretic OFKE Functionals}

Two papers $[113,114]$ put forth a novel and intriguing information-theoretic orbitalfree form for the zero-T KE functional (see the chapters of Hamilton and Delle Site for related developments). Its ancestry lies in the Sears-Parr-Dinur analysis [51]. Consider the $N_{e}$-fermion wave function and write

$$
N_{e} \Psi^{*}\left(\mathbf{r}_{1} \ldots \mathbf{r}_{N_{e}}\right) \Psi\left(\mathbf{r}_{1} \ldots \mathbf{r}_{N_{e}}\right)=n\left(\mathbf{r}_{1}\right) f\left(\mathbf{r}_{2} \ldots \mathbf{r}_{N_{e}} \| \mathbf{r}_{1}\right)
$$

This decomposition isolates the density, which is the identifier of the equivalence classes of states in the Levy-Lieb constrained search formulation of the universal functional $[2,3]$. Thus one can, for example, do constrained search Monte Carlo calculations [115] on the conditional factor $f\left(\mathbf{r}_{2} \ldots \mathbf{r}_{N_{e}} \| \mathbf{r}_{1}\right)$. The total KE then is

$$
T[\Psi]=\frac{1}{8} \int d \tau_{N_{e}} \frac{\left|\nabla_{1} n f\right|^{2}}{n f}=T_{W}[n]+\frac{1}{8} \int d \mathbf{r}_{1} d \tau_{N_{e}-1} \frac{\left|\nabla_{1} f\left(\tau_{N_{e}-1} \| \mathbf{r}_{1}\right)\right|^{2}}{f\left(\tau_{N_{e}-1} \| \mathbf{r}_{1}\right)} .
$$

(The space $\tau_{N_{e}-1}$ is compact notation for all the electron coordinates but $\mathbf{r}_{1}$.) In [115] a model form for $f\left(\tau_{N_{e}-1}\right)$ was constructed by considering necessary conditions on the $N_{e}$-fermion wave function in conjunction with Monte Carlo sampling on the homogeneous electron gas over a finite range of comparatively high (in our experience) densities $0.55 \leq r_{s} \leq 1.81$. The result was a proposed KE functional which, in our notation, is

$$
T_{G D S 08}[n]=T_{W}[n]+\int d \mathbf{r} n(\mathbf{r})\left[A_{1}+B_{1} \ln n(\mathbf{r})\right]
$$

with $A_{1}=0.860 \pm 0.022$ and $B_{1}=0.224 \pm 0.012$. A later, more refined version of the trial conditional probability function modeled the behavior of high-density fermion pairing [114]. Monte Carlo sampling gave what we called the GHDS10 functional,

$$
T_{G H D S 10}[n]=T_{W}+T_{T F}+\int d \mathbf{r} n(\mathbf{r})\left[A_{2}+B_{2} \ln n(\mathbf{r})\right],
$$

with $A_{2}=1.02$ and $B_{2}=0.163$. Both functionals have an evident informationtheoretic structure.

As proposed, there is an unfortunate flaw in both these functionals if they are used in the universal sense, namely they violate various positivity constraints outside certain density ranges [116]. The analysis in [116] led to a proposed modified information-theoretical form that also is positive definite. The challenge is how to implement that modified form, since it relies on the use of a maximal bounding function for the density. The examples given in [116] suffice for the mathematical purposes of that paper but are not practical. A related result is that the TF functional can be written

$$
T_{T F}=c_{T F} \int d \mathbf{r}\left(n+\frac{2}{3} n \ln n\right)+\frac{2}{3} c_{T F} \int d \mathbf{r} n \ln n \mathscr{L}[n] .
$$


with $\mathscr{L}[n]:=\left(2 c_{T F} / 3\right) \int d \mathbf{r} n \ln n \sum_{j=1}^{\infty} \frac{1}{(j+1) !}\left(\frac{2}{3} \ln n\right)^{j}$. With this, the von Weizsäcker plus parametrized Thomas-Fermi model,

$$
T_{W T F}[n]=T_{W}[n]+\gamma T_{T F}[n], 0 \leq \gamma \leq 1 .
$$

can be rewritten as

$$
\begin{aligned}
T_{W T F} & =T_{W}+\gamma_{c_{T F}}\left\{N_{e}+\frac{2}{3} \int d \mathbf{r} n \ln n\right\}+\frac{2}{3} \gamma_{T F} \int d \mathbf{r} n \ln n \mathscr{L}[n] \\
& =T_{G D S 08}+\Delta T .
\end{aligned}
$$

This result is suggestive of a possible route to an improved functional. One would combine Monte Carlo data with formal constraints so that the last line would be replaced, for example, by an improvement on $T_{G D S 08}$ such that the $\Delta T$ piece would be minimized in some sense (e.g., for a class of densities). How to give these vague but appealing ideas a useful form is not apparent to us at this time. Notice also the explicit $N_{e}$-dependence in (47), a reminder of our earlier discussion.

\subsection{Scale Function and Local-scaling DFT}

Uniform scaling, $\mathbf{r} \rightarrow \lambda \mathbf{r}$, with $\lambda$ a constant, is much-used in DFT to establish formal properties of density functionals via the scaled density $n_{\lambda}(\mathbf{r})=\lambda^{3} n(\lambda \mathbf{r})$ [117]. Local-scaling transformation (LST) DFT [118-120] instead exploits non-uniform, isotropic transformations

$$
\mathbf{r} \rightarrow \mathbf{g}(\mathbf{r}):=\lambda(\mathbf{r}) \mathbf{r}
$$

where $\lambda(\mathbf{r})$ is a local-scaling function. LST generates a set of density-dependent orbitals from an arbitrary initial set $\left\{\psi_{i}(\mathbf{r})\right\}_{i=1}^{N_{e}}$ by means of a unitary operation $\hat{\mathscr{U}}_{g}$

$$
\phi_{i}([n] ; \mathbf{r})=\hat{\mathscr{U}}_{g} \psi_{i}(\mathbf{r}):=[J(\mathbf{g}(\mathbf{r}) ; \mathbf{r})]^{1 / 2} \psi_{i}(\mathbf{g}(\mathbf{r})) .
$$

Here $J$ is the Jacobian of the transformation

$$
J(\mathbf{g}(\mathbf{r}) ; \mathbf{r})=\operatorname{det}\left[\frac{\partial g_{i}}{\partial r_{j}}\right]=\frac{n(\mathbf{r})}{n_{\psi}(\mathbf{g}(\mathbf{r}))},
$$

with $i, j=\{x, y, z\}$ and $n_{\psi}=\sum_{i} f_{i}\left|\psi_{i}\right|^{2}$. The KE corresponding to the set of orbitals Eq. (49) is a functional of the density $[120,121]$

$$
T_{s}\left[n,\left\{\psi_{i}\right\}_{i=1}^{N_{e}}\right]=T_{W}[n]+\frac{1}{2} \int d \mathbf{r} n^{5 / 3}(\mathbf{r}) A_{N_{e}}([n] ; \mathbf{r}),
$$

with parametric dependence on the initial set of orbitals $\left\{\psi_{i}(\mathbf{r})\right\}_{i=1}^{N_{e}}$. $A_{N_{e}}$ clearly is a Pauli term enhancement factor, Eq. (19), constructed in terms of the density $n$, transformed variable $\mathbf{g}$, and the initial orbital set $\{\psi\}$ [118].

Interestingly, Xia and Carter [63] use a scalar local scaling function $F$ for a different purpose, namely to decompose the density into delocalized and localized 
parts

$$
n(\mathbf{r})=n_{d e l}(\mathbf{r})+n_{l o c}(\mathbf{r}):=F([n] ; \mathbf{r}) n(\mathbf{r})+(1-F([n] ; \mathbf{r})) n(\mathbf{r}) .
$$

This decomposition, together with a simple model for the localized KE (parametrized Thomas-Fermi plus von Weizsäcker) enables them to use the two-point WangGovind-Carter (WGC) functional $[122,123]$ for the delocalized density alone. The WGC functional is well-adapted to that type of density. Thus, Xia and Carter construct a broadly useful two-point KE functional.

In the Euler-Lagrange equation for their functional, Xia and Carter neglect the functional derivative $\delta F / \delta n$, although the form used clearly is a density functional (it is tabulated numerically with the ratio of the density to the average delocalized density as the argument). Instead, they treat $F$ as a simple function of $\mathbf{r}$ and evaluate its density dependence by introducing an extra self-consistent loop. The effect is that they reach an $F$ for which $\delta F / \delta n \approx \Delta F / \Delta n=0$, with the finite increments being iteration-to-iteration differences in the extra loop. Clearly their solution is special, since their functional does not have zero functional derivative in general. We have devised an analytical $F$ which resembles the Xia-Carter form but uses a fixed reference delocalized density. Thus it is easy to differentiate functionally. At this writing we have just begun to explore it. More broadly, one may contemplate reverse engineering of the Xia-Carter KE functional to uncover the local scaling transformation to which it corresponds.

\section{A Few of the Challenges Ahead}

One of the most difficult problems faced by the orbital-free agenda at present is the XC functional. From both the work with our collaborators in México as well as others (e.g., [89] and references therein), it is clear that simple GGA forms do not suffice. Broadly, in simple GGAs for XC what betters molecular predictions worsens those for solids. Whether some quite complicated GGA form exists that is equally good for both system types is unknown. The conventional wisdom seems to be that it does not. But the arguments behind that conventional wisdom tend to rely either on the operational failure of simple forms (the molecular properties versus solid properties issue) or on the observation that there are rigorous constraints on the exact $E_{x c}$ which give contradictory requirements on a GGA. An example is the contradictory limiting behavior $\lim _{s \rightarrow \infty} F_{x}^{G G A}(s)$ discussed in [72, 84]. But such contradictions so far have not impeded the development of better functionals because it has been possible to determine which constraints are relevant to realsystem properties.

On the KE functional side, we already have mentioned the embarrassment of riches challenge posed by the reduced density derivatives which suppress, at least to some order in the gradient expansion, nuclear site singularities in $\mathrm{v}_{\theta}$. We reiterate the challenge here. In [67] there is a set of RDDs which are candidates for indepen-

dent variables in beyond-GGA OFKE functionals. Since the paper by Perdew and Constantin [124] on a meta-GGA OFKE functional and our own work [67], there has 
been little progress on conditions and constraints for constructing RDD-dependent OFKE functionals. Although Perdew and Constantin parallel, broadly speaking, the route from a GGA to a meta-GGA for XC, it is not clear that the approach is best. In a GGA for the OFKE, there is only one variable $s$, so the effect of exact constraints can be worked out comparatively straightforwardly. Since there are many higher-level RDDs, it appears that one must select some subset of them, then work out the consequences of exact constraints for a general enhancement which depends on that specific set of RDDs. Perdew and Constantin [124] picked $s$ and a reduced Laplacian, for example. Any choice, of course, leads immediately to the issue of the criteria. We have no new, substantive insight on that at the moment.

A different possible route forward is that all current OF-DFT functionals fail to satisfy the differential virial relationship between the KE and XC functional [117]. In principle, OFKE and OFXC functionals should be paired. We currently are investigating whether a useful RDD-dependent OFKE functional can be generated from a reasonably good GGA XC functional.

Finally, back at the refinement level of GGA OFKE functionals, now that we have the breakthrough of one non-empirical parametrization of a properly positive definite functional [80], there is an opportunity to improve that scheme. Almost certainly it is not optimal. Again, routes to improvement are not totally obvious.

Acknowledgements We acknowledge, with thanks, the many contributions and influences especially of Travis Sjostrom and of our other primary collaborators, Jim Dufty, Frank Harris, Keith Runge, Alberto Vela, Jose Luis Gázquez, and Jorge Martín del Campo. Thanks also go to Emilio Orgaz, Victor Medel, Támas Gál, and J.C. Pacheco-Kato. Helpful conversations with P. Ayers, K. Burke, K. Capelle, E.A. Carter, L. Delle Site, D. Gárcia-Aldea, A. Görling, M. Levy, M.M. Odashima, J.P. Perdew, N. Rösch, A. Ruzsinszky, and T. Wesolowski are acknowledged with tharsks. We thank Luigi Delle Site and Volker Bach for the opportunity to participate in this volume and for the opportunity provided to SBT to attend the NAMET meeting in 2010. This work was supported under U.S. Dept. of Energy BES grant DE-SC 0002139.

\section{References}

1. P. Hohenberg and W. Kohn, Phys. Rev. 136, B864 (1964).

2. M. Levy, Proc. Natl. Acad. Sci. USA 76, 6062 (1979).

3. E.H. Lieb, Int. J. Quantum Chem. 24, 243 (1983)

4. L.H. Thomas, Proc. Camb. Phil. Soc. 23, 542 (1927).

5. E. Fermi, Rend. Accad. Naz. Lincei 6, 602 (1927).

6. E. Fermi, Z. Phys. 48, 73 (1928).

7. P.A.M. Dirac, Proc. Cambridge Phil. Soc. 26, 376 (1930).

8. C.F. von Weizsäcker, Z. Phys. 96, 431 (1935).

9. E. Teller, Rev. Mod. Phys. 34, 627 (1962).

10. E.H. Lieb, Rev. Mod. Phys. 53, 603 (1981).

11. W. Kohn and L.J. Sham, Phys. Rev. 140, A1133 (1965).

12. J.C. Boettger and S.B. Trickey, Phys. Rev. B 51, 15623(R) (1995).

13. J.C. Boettger and S.B. Trickey, Phys. Rev. B 53, 3007 (1996).

14. R.N. Barnett and U. Landman, Phys. Rev. B 48, 2081 (1993).

15. D. Marx and J. Hutter, in Modern Methods and Algorithms of Quantum Chemistry, J. Grotendorst, ed., J. von Neumann Inst. for Computing, (Jülich, NIC Series, Vol. 1, 2000) p. 301 and refs. therein.

16. J.S. Tse, Annu. Rev. Phys. Chem. 53, 249 (2002). 
17. D. Marx and J. Hutter, Ab Initio Molecular Dynamics: Basic Theory and Advanced Methods (Cambridge University Press, Cambridge, 2009) and refs. therein.

18. T.D. Kühne,arXiv: 1201.5945.

19. R.G. Parr and W. Yang, Density Functional Theory of Atoms and Molecules (Oxford, NY, 1989).

20. R.M. Dreizler and E.K.U. Gross, Density Functional Theory (Springer-Verlag, Berlin, 1990).

21. A.D. Becke, J. Chem. Phys. 98, 1372 (1993).

22. P.J. Stephens, F.J. Devlin, C.F. Chabalowski, and M.J. Frisch, J. Phys. Chem. 98, 11623 (1994).

23. A. Görling and M. Levy, J. Chem. Phys. 106, 2675 (1997).

24. A. Görling, Phys. Rev. B 53, 7024 (1996); erratum, ibid. 59, 10370 (1999).

25. S. Ivanov, S. Hirata, and R.J. Bartlett, Phys. Rev. Lett. 83, 5455 (1999).

26. J.P. Perdew and K. Schmidt, A.I.P. Conf. Proc. 577, 1 (2001).

27. E. Engel in A Primer in Density Functional Theory, C. Fiolhais, F. Nogueira, and M.A.L. Marques eds. (Springer, Berlin, 2003) p. 56 and refs. therein.

28. J. Heyd, G.E. Scuseria, and M. Ernzerhof, J. Chem. Phys. 118, 8207 (2003); erratum, ibid. 124, 219906 (2006).

29. A.V. Krukau, O.A. Vydrov, A.F. Izmaylov, and G.E. Scuseria, J. Chem. Phys. 125, 224106 (2006).

30. A. Heßelmann, A.W. Götz, F. Della Sala, and A. Görling, J. Chem. Phys. 127, 054102 (2007).

31. S. Kümmel and L. Kronik, Rev. Mod. Phys. 80, 3 (2008) and refs. therein.

32. V.V. Karasiev, and E.V. Ludeña, Phys. Rev. A 65, 062510 (2002).

33. I. González, C. González, V.V. Karasiev, E.V. Ludeña, and A.J. Hernández, J. Chem. Phys. 118, 8161 (2003).

34. V.V. Karasiev, J. Chem. Phys. 118, 8576 (2003).

35. T. Grabo, T. Kreibach, S. Kurth, and E.K.U. Gross in Strong Coulomb Correlations in Electronic Structure: Beyond the Local Density Approximation, V.I. Anisimov ed. (Gordon and Breach, Tokyo, 2000) p. 203 and refs. therein.

36. J. Clerouin, E.L. Pollock, and G. Zerah, Phys. Rev. A 46, 5130(1992)

37. E. Chacón, J.E. Alvarellos, and P. Tarazona, Phys. Rev. B 32, 7868 (1985).

38. L.W. Wang and M.P. Teter, Phys. Rev. B 45, 13196(1992).

39. M. Foley, E. Smargiassi and P.A. Madden, J. Phys.: Condens. Matt. 6, 5231(1993).

40. M. Pearson, E. Smargiassi and P.A. Madden, J. Phys.: Condens. Matt. 5, 3221(1993).

41. E. Smargiassi and P.A. Madden, Phys. Rev. B 49, 5220(1994).

42. M.D. Glossman, L.C. Bálbas, A. Rubio, and J.A. Alonso, Int. J. Quantum Chem. 49, 171 (1994).

43. V.V. Karasiev, T. Sjostrom, D. Chakraborty, J.W. Dufty, F.E. Harris, K. Runge, and S.B. Trickey, in Computational Challenges in Warm Dense Matter, F. Graziani et al. eds. (Springer Verlag, Heidelberg) in press.

44. T.A. Wesołowski, Phys. Rev. A 77, 012504 (2008) and refs. therein.

45. J.W. Kaminski, S. Gusarov, T.A. Wesołowski, and A. Kovalenko, J. Phys. Chem. A 114, 6082 (2010) and refs. therein.

46. J.P. Perdew, K. Burke, and M. Ernzerhof, Phys. Rev. Lett. 77, 3865 (1996); erratum ibid. 78, 1396 (1997).

47. J.P. Perdew and A. Zunger, Phys. Rev. B 23, 5048 (1981).

48. A.C. Cancio, C.E. Wagner, and S.A. Wood, Int. Quant. Chem. 112, 3796 (2012).

49. SBT is indebted to So Hirata for this observation (2005).

50. M. Hoffmann-Ostenhof and T. Hoffmann-Ostenhof, Phys. Rev. A 16, 1782 (1977); see Eq. (2.20). Also see E. Romera and J.S. Dehesa, Phys. Rev. A 50, 256 (1994).

51. S.B. Sears, R.G. Parr, and U. Dinur, Isr. J. Chem. 19, 165 (1980).

52. J.E. Harriman, J. Chem. Phys. 83, 6283 (1985).

53. C. Herring, Phys. Rev. A 34, 2614 (1986).

54. M. Levy, J.P. Perdew, and V. Sahni, Phys. Rev. A 30, 2745 (1984).

55. Y. Tal and R.F.W. Bader, Int. J. Quantum Chem. S12, 153 (1978).

56. L.J. Bartolotti and P.K. Acharya, J. Chem. Phys. 77, 4576 (1982). 
57. J.E. Harriman, in Density Matrices and Density Functionals, R. Erdahl and V.H. Smith Jr. eds. (D. Reidel, Dordrecht, 1987), p. 359.

58. M. Levy and H. Ou-Yang, Phys. Rev. A 38, 625 (1988).

59. R. Baltin, J. Chem. Phys. 86, 947 (1987).

60. Y.A. Wang and E.A. Carter, Chap. 5 in Theoretical Methods in Condensed Phase Chemistry, S.D. Schwartz, ed. (Kluwer, NY 2000), p. 117 and refs. therein.

61. B.J. Zhou, V.L. Lignères, and E.A. Carter, J. Chem. Phys. 122, 044103 (2005).

62. C. Huang and E.A. Carter, Phys. Rev. B 81, 045206 (2010).

63. J. Xia and E.A. Carter, Phys. Rev. B 86, 235109 (2012).

64. M. Hodak, W. Lu, and J. Bernholc, J. Chem. Phys. 128, 014101 (2008).

65. X. Blanc and E. Cances, J. Chem. Phys. 122, 214106 (2005).

66. Y. Zhao and D G. Truhlar, J. Chem. Phys. 125, 194101 (2006).

67. V.V. Karasiev, R.S. Jones, S.B. Trickey, and F.E. Harris, Phys. Rev. B 80, 245120 (2009).

68. V.V. Karasiev, S.B. Trickey, and F.E. Harris, J. Computer-Aided Mat. Design, 13, 111 (2006).

69. V.V. Karasiev, R.S. Jones, S.B. Trickey, and F.E. Harris, in New Developments in Quantum Chemistry, J.L. Paz and A.J. Hernández, eds. (Transworld Research Network, Kerala, India, 2009) p. 25.

70. S.B. Trickey, V.V. Karasiev, and R.S. Jones, Int. J. Quantum Chem. 109, 2943 (2009).

71. A. Vela, V. Medel, and S.B. Trickey, J. Chem. Phys. 130, 244103 (2009);

72. A. Vela, J.C. Pacheco-Kato, J.L. Gázquez, J.M. del Campo, and S.B. Trickey, J. Chem. Phys. 136, 144115 (2012).

73. J.P. Perdew, Phys. Lett. A 165, 79 (1992).

74. D.J. Lacks and R.G. Gordon, J. Chem. Phys. 100, 4446 (1994).

75. A.E. DePristo and J.D. Kress, Phys. Rev. A 35, 438 (1987).

76. A.J. Thakkar, Phys. Rev. A 46, 6920 (1992).

77. F. Tran and T.A. Wesołowski, Int. J. Quantum Chem. 89, 441 (2002).

78. V.V. Karasiev and S.B. Trickey, Comput. Phys. Commun. 183, 2519 (2012).

79. A. Borgoo and D.J. Tozer, J. Chem. Theory Comput. doi 10.1021/ct400129d (2013).

80. V.V. Karasiev, D. Chakraborty, O.A. Shukruto, and S.B. Trickey, arXiv 1308.2193.

81. C.H. Hodges, Can. J. Phys. 51, 1428 (1973).

82. T. Kato, Commun. Pure Appl. Math. 10, 151 (1957).

83. J.M. del Campo, J.L. Gázquez, R.J. Alvarez-Mendez, S.B. Trickey, and A. Vela, in Concepts and Methods in Modern Theoretical Chemistry, Vol. 1, S.K. Ghosh and P.K. Chattaraj eds. (CRC Press, Boca Raton, 2013) p. 295 and refs. therein.

84. J.M. del Campo, J.L. Gázquez, S.B. Trickey, and A. Vela, J. Chem. Phys. 136, 104108 (2012).

85. J.M. del Campo, A. Vela, J.L. Gázquez, and S.B. Trickey, Chem. Phys. Lett. 543, 179 (2012).

86. J.P. Perdew, A. Ruzsinszky, G.I. Csonka, L.A. Constantin, and J.W. Sun, Phys. Rev. Lett. 103, 026403 (2009). erratum ibid. 106, 179902 (2011).

87. E.H. Lieb and S. Oxford, Int. J. Quantum Chem. 19, 427 (1981); E.H. Lieb, Phys. Lett. 70A, 444 ( 1979).

88. J.P. Perdew, A. Ruzsinszky, G.I. Csonka, O.A. Vydrov, G.E. Scuseria, L.A. Constantin, X.L. Zhou, and K. Burke, Phys. Rev. Lett. 100, 136406 (2008).

89. P. Haas, F. Tran, P. Blaha, L.S. Pedroza, A.J.R. da Silva, M.M. Odashima, and K. Capelle, Phys. Rev. B 81, 125136 (2010).

90. G.K-L. Chan, A.J. Cohen, and N.C. Handy, J. Chem. Phys. 114, 631 (2001).

91. V.V. Karasiev, T. Sjostrom, and S.B. Trickey, Phys. Rev. E 86, 056704 (2012).

92. X. Gonze, B. Amadon, P.-M. Anglade, J.-M. Beuken, F. Bottin, P. Boulanger, F. Bruneval, D. Caliste, R. Caracas, M. Cote, T. Deutsch, L. Genovese, Ph. Ghosez, M. Giantomassi, S. Goedecker, D.R. Hamann, P. Hermet, F. Jollet, G. Jomard, S. Leroux, M. Mancini, S. Mazevet, M.J.T. Oliveira, G. Onida, Y. Pouillon, T. Rangel, G.-M. Rignanese, D. Sangalli, R. Shaltaf, M. Torrent, M.J. Verstraete, G. Zerah, and J.W. Zwanziger, Comput. Phys. Commun. 180, 2582 (2009);

93. X. Gonze, G.-M. Rignanese, M. Verstraete, J.-M. Beuken, Y. Pouillon, R. Caracas, F. Jollet, M. Torrent, G. Zerah, M. Mikami, Ph. Ghosez, M. Veithen, J.-Y. Raty, V. Olevano, F. Bruneval, L. Reining, R. Godby, G. Onida, D.R. Hamann, and D.C. Allan, Zeit. Kristallogr. 220, 558 (2005). 
94. P. Giannozzi, S. Baroni, N. Bonini, M. Calandra, R. Car, C. Cavazzoni, D. Ceresoli, G.L. Chiarotti, M. Cococcioni, I. Dabo, A. Dal Corso, S. de Gironcoli, S. Fabris, G. Fratesi, R. Gebauer, U. Gerstmann, C. Gougoussis, A. Kokalj, M. Lazzeri, L. Martin-Samos, N. Marzari, F. Mauri, R. Mazzarello, S. Paolini, A. Pasquarello, L. Paulatto, C. Sbraccia, S. Scandolo, G. Sclauzero, A.P. Seitsonen, A. Smogunov, P. Umari, and R.M. Wentzcovitch, J. Phys.: Condens. Matt. 21, 395502 (2009).

95. G. Kresse and J. Hafner, Phys. Rev. B 47, 558 (1993).

96. G. Kresse and J. Hafner, Phys. Rev. B 49, 14251 (1994).

97. G. Kresse and J. Furthmüller, Comput. Mat. Sci. 6, 15 (1996).

98. G. Kresse and J. Furthmüller, Phys. Rev. B 54, 11169 (1996).

99. V. Heine and I. Abarenkov, Phil. Mag. 9, 451 (1964).

100. L. Goodwin, R.J. Needs, and V. Heine, J. Phys.: Condens. Matt. 2, 351 (1990).

101. V.V. Karasiev, T. Sjostrom, and S.B. Trickey, Phys. Rev. B 86, 115101 (2012).

102. H.Y. Geng, H.X. Song, J.F. Li, and Q. Wu, J. Appl. Phys. 111, 063510 (2012).

103. G.S. Ho, V.L. Lignères, and E.A. Carter, Comput. Phys. Commun. 179, 839 (2008).

104. L. Hung, C. Huang, I. Shin, G.S. Ho, V.L. Lignères, and E.A. Carter, Comput. Phys. Commun. 181, 2208 (2010).

105. V.V. Karasiev, T. Sjostrom, and S.B. Trickey, "Orbital-free Density Functional Molecular Dynamics: Coupling PROFESS and QUANTUM ESPRESSO", in preparation.

106. L.A. Constantin, E. Fabiano, S. Laricchia, and F. Della Sala, Phys. Rev. Lett. 106, 186406 (2011).

107. B. Wang, M.J. Stott, and U. von Barth, Phys. Rev. A 63, 052501 (2001).

108. S.B. Trickey and A. Vela, J. Mex. Chem. Soc. 57, 105 (2013).

109. P.K. Acharya, L.J. Bartolotti, S.B. Sears, and R.G. Parr, Proc. Natl. Acad. Sci. (USA) 77, 6978 (1980).

110. W. Yang, R.G. Parr, and C. Lee, Phys. Rev. A 34, 4586 (1986).

111. D. Gárcia-Aldea and J.E. Alvarellos, J. Chem. Phys. 127, 144109 (2007).

112. D. Gárcia-Aldea and J.E. Alvarellos, Phys. Chem. Chem. Phys. 14, 1756 (2012).

113. L. Delle Site, Eur. Phys. Lett. 86, 40004 (2009); erratum ibid. 88, 19901 (2009).

114. L.M. Ghiringhelli, I.P. Hamilton, and L. Delle Site, J. Chem. Phys. 132, 014106 (2010),

115. L.M. Ghiringhelli and L. Delle Site, Phys. Rev. B 77, 073104 (2008).

116. S.B. Trickey, V.V. Karasiev, and A. Vela, Phys. Rev. B 84, 075146 (2011).

117. M. Levy and J.P. Perdew, Phys. Rev. A 32, 2010 (1985).

118. E.V. Ludeña and R. López-Boada, Top. Curr. Chem. 180, 169 (1996).

119. M.V. Stoitsov and I.Zh. Petkov, Annals Phys. 185, 121 (1988).

120. E.V. Ludeña, V. Karasiev, R. López-Boada, E. Valderrama, and J. Maldonado, J. Chem. Phys. 20, 155 (1999).

121. E.V. Ludeña and V.V. Karasiev, In: Reviews of Modern Quantum Chemistry: a Celebration of the Contributions of Robert Parr. K.D. Sen (Ed.), (World Scientific, Singapore, 2002) p. 612.

122. Y.A. Wang, N. Govind, and E.A. Carter, Phys. Rev. B 58, 13465 (1998); erratum ibid. 64, 129901 (2001).

123. Y.A. Wang, N. Govind, and E.A. Carter, Phys. Rev. B 60, 16350 (1999); erratum ibid. 64, 089903 (2001)

124. J.P. Perdew and L.A. Constantin, Phys. Rev. B 75, 155109 (2007). 